\title{
Anna Dutka-Mańkowska \\ Université de Varsovie \\ LE DISCOURS INDIRECT MIMÉTIQUE ET SA TRADUCTION DU FRANÇAIS EN POLONAIS DANS DES TEXTES LITTÉRAIRES
}

\section{Mimetic reported speech and its translation from French to Polish in literary texts}

\begin{abstract}
This article deals with the French-to-Polish translation of a specific case of reported speech: the investigated examples from literary texts are, from a semantic point of view, paraphrases by the narrator, who formulates the words of the characters in reported speech. They do not, however, only express his point of view (this is a form of reported speech called mixed or mimetic). In the Polish text, the discourse of the characters includes graphic, grammatical or lexical ant indicators, as well as the interpretation of statements in the context of the translation 's reader. A few important guidelines are given, on the basis of which the reader can interpret the passages in which the reported speech comes close to direct speech or to speech in a situation. Attention has also been paid to the modifications that the narrator's speech undergoes when their discourse creates the context of the speech of the character.
\end{abstract}

KEYWORDS: cited speech, mixed reported speech, translation, French, Polish, active text comprehension

\section{INTRODUCTION}

Le discours rapporté ${ }^{1}$ est présenté le plus souvent à partir des schémas syntaxiques qui distinguent la discours direct (DD), le discours indirect avec l'enchâssement (DI) et le style indirect libre (DIL) qui « combine les particularités du discours direct et indirect » (Riegel et al. 2009 : 1015). On admet implicitement qu'ils sont bien identifiables et distinctes les uns des autres et que les formes « sont productrices d'un sens, toujurs le même, le sens rapporté » (De Mattia-Viviès $2006: 29$ ).

Les approches sémantiques du discours rapporté, en référence à la linguistique d'énonciation, ont identifié une variété de formes, telles que $D D$ avec que, discours direct libre, les formes comme selon $X$, d'après $X$, les gloses métaénonciatives qui croisent le domaine du DR, telles que $X$, comme dit $Y$, la modalisation propre au conditionnel, les formes à la frontière du dire anonyme comme dit-on, on dit que, et d'autres.

1 Pour l'emploi des termes discours rapporté et discours représenté, voir Dutka-Mańkowska, Kostro (dir.) (2017: 7-12). 
Nous nous référons à deux grandes théories. Jacqueline Authier-Revuz (2004) pose deux modes de la représentation du discours autre: il est l'objet du dire ou bien il sert à modaliser le discours en train de se faire. Elle propose une conception métalinguistique du DR. L'auteure distingue, à l'instar d'É. Benveniste, le niveau de la langue et celui du discours, ou divers phénomènes se superposent à ceux de la langue. Au niveau de la langue, le DD, hétérogène (segment en usage + segment en mention) est distingué du DI, qui est une paraphrase homogène du point de vue du rapporteur et donne d'image du discours autre par une paraphrase discursive. C'est le rapporteur qui ramène le discours autre au sien. Il s'agit surtout (Authier-Revuz 1993 : 13-14) de la désignation par descriptions définies, les éléments expressifs et exclamatifs, les évaluatifs et les « manières de dire », le DI avec îlot textuel et le DI quasi-textuel. Les formes mixtes (le mélange du DD et du DI) sont exclues au niveau des formes de langue. Laurence Rosier plaide, au contraire, pour les formes mixtes ${ }^{2}$ qui possèdent la typographie du DD, mais les marques grammaticales du DI et elle propose de systématiser le champ du DR en continuum (Rosier 2008). Seulement le DIL a été parfois envisagé comme une forme mixte. Mais on peut avoir des distinctions sémantiques sans marquage grammatical, qui en contexte font aussi reconsidérer les formes du DI comme non homogènes (p.ex. Jonasson 2002, Rosier 2006, De Mattia-Viviès 2010). Nous serons concernée par le plurilinguisme et par les formes de polyphonie lorsqu'elles se réalisent dans un discours rapporté (Rosier 2006 : 20). En termes de Bakhtine (1978 : 125-126), il s'agit de la bivocalité, caractéristique des énoncés qu'il appelle, dans le domaine littéraire, « constructions hybrides ». Ce sont des énoncés qui formellement se présentent comme appartenant à un seul locuteur, mais "deux perspectives sémantiques et sociologiques" s'y confondent. M. De Mattia-Viviès (2010 : 10) parle de la « syntaxe mensongère » qui n'affiche pas le DI hétérogène (Jonasson 2002 ). Le lecteur actualise les textes de fiction qui lui donnent l'illusion que les événements se déroulent sous ses yeux (Rosier 1999 : 151). L'hétérogénéité énonciative dans le DR s'oriente vers l'actualisation du discours cité (Dé) (Strauch cité par Rosier 1999 : 141). Ainsi le DI tend à se rapprocher du DD et du discours origine (discours en direct). La question des effets de réel est liée à celle de l'oralisation, que nous envisagerons ici d'une manière générale (voir Dutka-Mańkowska 2015), sans distinguer celle qui vise à restituer la parole vive du personage à l'écrit, comme chez Flaubert, de celle qui se propose d'introduire l'oral populaire dans un texte littéraire, comme chez Zola (G. Philippe cité par Wolf 2016). Les facteurs culturels sont fondamentaux: rappelons que verbatim est sensible aux facteurs culturels (Coulmas 1986) et le réalisme, p.ex. dans le dialogue romanesque, est fondé « sur les preéupposés pragmatiques, idéologiques et culturels attestés dans le hors-texte » (Lane-Mercier 1989 : 45-49). Ainsi certains glissements s'expliquent par des contraintes linguistiques, d'autres par le co-et contexte, finalement aussi par les normes littéraires et translationnelles (Poncharal 2003).

Dans la perspective traductologique, K. Taivalkoski-Shilov (2006:43) a employé le terme de discours indirect mimétique (DIM), un type mixte (ou hybride) du DR, avec des indices qui peuvent être grammaticaux (décalages déictiques ou temporels), lexicaux (termes dialectaux, étrangers, etc. placés dans le style neutre du narrateur), typographiques (guillemets, italiques, points de suspension...). La distinction entre le DD et le

2 «mixte signifie mélange de critères distinctifs (qui peut aller jusqu'à la rupture syntaxique) et il existe bien un discours indirect avec guillemets » (Rosier 1999 : 113). 
DI en français est forte, ainsi le cas des mentions hybrides, mélanges ou mixtes (Rosier 1999 : 245) est particulièrement intéressant du point de vue de la traduction. Comment le texte traduit (TA) permet-il au lecteur polonais de reconstruire l'effet de l'actualisation du discours cité dans le DI, si le français "semble privilégier le point de vue englobant du rapporteur au détriment du locuteur initial" (Taivalkoski-Shilov $2006: 69$ ) ?

En référence à Alain Rabatel (2008), nous admettons une relation active du lecteur par rapport au texte, qui comporte des instructions quant à la manière de s'approprier le texte, dans notre cas, d'un texte traduit. Les textes littéraires sont orientés vers la création et ils supposent un lecteur compétent.

\section{LA RESTITUTION DE LA PAROLE CITÉE DANS SON ÉNONCIATION DIRECTE}

\subsection{Le niveau grammatical : l'absence de la concordance des temps en polonais}

En français les temps, les pronoms et les adverbes déictiques varient si l'énonciation est homogène, en DI et en discours narrativisé. Le DD conserve un double système de repérages. Le système temporel polonais n'obéit pas au principe de la transposition et il permet l'actualisation du Dé :

(1) Le portier confidentiel voulut en vain le persuader que « ce nouveau système sans faire de vent absorbait la fumée », (Mauriac fr 737)

(1') Usłużny portier usiłował na próżno przekonać go, że "ten nowy system wchłania dym, nie robiąc wiatru". (Mauriac pl 5)

L'emploi de la forme passée dans la proposition principale construit un axe temporel. Tous les procès de la proposition subordonnée s'inscrivent sur cet axe sans une forme spécialisée (Tomaszkiewicz 1988 : 85). C'est aussi le cas du présent dans la complétive. Pour cette raison, il n'est pas possible de rendre l'hétérogénéité du DI propre au texte de depart (TD) lorsque le verbe est justement au présent (comme ci-dessous le verbe $i l$ faut) :

(2) Le prêtre [...] lança du haut de la chair des imprécations, des malédictions et des menaces contre le château, disant qu'il faut porter le fer rouge dans les plaies, anathémisant le baron qui s'en amusa, [...] (Maupassant fr 173)

(2') Mówił o wrzodach, które trzeba wypalić żelazem, złorzeczył baronowi, którego to bawiło, [...] (Maupassant pl 144)

Le présent dans le TA, qui s'interprète comme une actualisation du Dé, peut s'accompagner d'un déictique au lieu d'une expression anaphorique (jutro 'demain' au lieu de nazajutrz 'le lendemain'):

(3) [...] pendant le dîner il [Swann] venait de dire que le lendemain il avait un banquet d'anciens camarades [...] (Proust fr 271)

(3’) Swann powiedział przy stole, że jutro ma jakiś koleżeński bankiet [...] (Proust pl 313) 


\subsection{Les termes de désignation}

Le Dé peut se manifester par des expressions compatibles avec la situation d'allocution d'origine, dans les deux langues:

(4) Quand elle rentra, le soir, on lui dit qu'un homme l'avait demandée de la part de M. Paul [...] (Maupassant fr 189)

(4') Kiedy wróciła wieczorem do hotelu, powiedziano jej, że pytał o nią jakiś człowiek przysłany przez pana Pawła [...] (Maupassant 189)

À la réception, un employé de l'hôtel a pu reprendre une forme familière du Dé. En polonais on a un équivalent fidèle, mais le statut en est différent: il est fréquent dans les situations informelles, p.ex. on s'adresse à une personne connue, qu'on vouvoie, avec le prénom précédé par pani 'madame'/ panie 'monsieur' (pani Mario 'madame Marie').

(5) et madame Tuvache [...] déclara devant sa servante que madame Bovary se compromettait. (Flaubert fr 160)

(5') a pani Tuvache [...] orzekła nawet, i to przy służącej, że doktorowa się kompromituje. (Flaubert E 111)

(5”) a pani Tuvache [...] oświadczyła swojej służącej, że pani Bovary kompromituje się. (Flaubert M : 69)

La désignation Madame Bovary est rendue dans (5') par une forme familière ancrée dans la morphologie: le suffixe -owa désigne d'une manière familière la femme d'un médecin (doktorowa), d'un président (prezydentowa), d'un ingénieur (inżynierowa), d'un professeur (profesorowa). (SJP PWN, dont elle rend le statut social, et non le nom de femme mariée.) Cette substitution dans le TA se fait en fonction de savoirs supposés du lecteur (voir Maingueneau 1994 : 128).

\subsection{Les éléments expressifs et exclamatifs}

Le maintien de ces éléments est aléatoire et résulte des moyens linguistique différents. Voici un renforcement de l'expressivité par un lexème :

(6) mais, quand elle lui disait qu'il faut aimer, beaucoup, beaucoup le bon Dieu, il répondait parfois: (Maupassant fr 182)

(6') lecz dowiadując się, że trzeba bardzo, bardzo kochać dobrą Bozię, pytał czasem: (Maupassant pl 154)

Bozia est un diminutif de Dieu, dans le langage des enfants, et l'énoncé reprend les paroles de la mère adressées à son fils.

Dans $\left(7^{\prime}\right)$, au contraire, on observe la réduction de la modalité exclamative, et sa conservation dans ( 7 "). Cet aspect de la construction du TA montre la proximité du traducteur avec le narrateur dans la construction du contexte du Dé :

(7) Mais, à tout cela, M. Bovary, peu soucieux des letters, disait que ce n'était pas la peine! (Flaubert 61) 
(7') Ale pan Bovary lekceważył sztuki piękne, mówił, że "na nic się to wszystko nie zda". (F1 M 8)

(7’) Ale pan Bovary, który lekceważył literaturę, mówił, że szkoda zachodu! (F1 E 35)

Les marques graphiques donnent une consigne univoque sur les frontières du Dé qui se présente comme un îlot textuel (Authier-Revuz 1993).

Le déterminant démonstratif signale une qualification nouvelle du nom, c'est un subjectivème. Pour D. Leemann (2004), il marque un point de vue personnel et comporte une instruction de créer un espace dans lequel son référent est situé: $\mathrm{Ce} N=l e N+i c i$ + rupture. Il peut véhiculer l'expressivité, comme dans (8). L'effacement dans (8') la réduit, mais il faut tenir compte de l'usage moins étendu des démonstratifs en polonais par rapport au français (Dutka-Mańkowska 2016), donc d'une tendance collective, et non des décisions individuelles d'un traducteur :

(8) Elle s'était remise à parler haut, s'emportant, s'indignant de ces intérêts négligés, de cette ruine menaçante. (Maupassant fr 197)

(8') Znów podniosła głos, unosząc się oburzeniem na myśl o tych nie zapłaconych procentach i o grożącej ruinie. (Maupassant pl 170)

\subsection{Les manières de parler}

D'autres moyens peuvent être pertinents afficher une caractéristique du Dé au sein de la paraphrase du narrateur.

Les guillements et les italiques se conservent pour délimiter son étendue (ex. 1-1', $5-5,7-7$ '-7', 12-12'), ou bien ils disparaissent de la traduction (ex. 5'), et ce sont les lèxèmes ou le contexte qui fondent l'interprétation. Voyons trois exemples de la construction de l'altérité par le lecteur:

(9) Puisque la maîtresse me « reprenait », plus tard j'ai voulu reprendre mon père, lui annoncer que « se parterrer » ou « quart moins onze heures » n'existaient pas. (Ernaux fr 64)

(9') Ponieważ nauczycielka mnie poprawiała, później ja zapragnęłam poprawiać ojca, pouczając go, że nie ma takiego słowa jak "szturgać" czy wyrażenia "kwadrans na jedenastą". (Ernaux pl 33)

L'italique final déclenche l'interprétation. Dans le contexte de la qualification reprendre, il nous semble suggérer une manière de parler de l'institutrice, peut-être elle martelait les mots (en italique). Le fragment rendrait compte d'une relation hiérarchique entre l'élève et sa professeure qui reprend (syn. réprimande, blame, critique, corrige) la fille. La marque graphique et l'ordre des mots sont à prendre ensemble. En ( $\left.7^{\prime}\right)$ on a une trace de la critique, le verbe pouczać 'faire la leçon' pour annoncer. Mais le texte traduit ne signale pas le rôle de la prosodie.

Dans (10) la manière de parler se fonde sur la maîtrise de la parole, attestée par un énoncé long. Il présente des promesses faites à une mère désespérée d'un fils atteint d'hémophilie, de la mettre en contact avec un spécialiste, par une chaîne d'intermédiaires : 
(10) on lui avait promis de lui faire rencontrer quelqu'un qui pouvait peut-être lui faire connaître un grand spécialiste des maladies du sang qui pourrait probablement... (Makine fr 88)

(10') obiecywano jej spotkanie z kimś, kto podobno zna wybitnego specjalistę od chorób krwi, z kimś, kto mógłby ewentualnie... (Makine pl 77)

La segmentation de l'énoncé $\left(10^{\prime}\right)$ réduit le nombre de personnes à contacter et gomme le caractère douteux des promesses creuses qui s'enchaînent.

Lorsqu'un domaine, comme la religion, offre en polonais des expressions parallèles à celles françaises, une manière de parler peut se conserver :

(11) Il lui promit un demi-pardon, Dieu ne pouvant déverser toutes ses grâces sur un toit qui recouvrait un homme comme le baron : [...] (Maupassant fr 169)

(11') Pocieszył ją, że Bóg odpuści jej winy, choć tylko częściowo, bo nie może On zsyłać pełni swych lask na dom, pod którego dachem żyje taki człowiek jak baron. (Maupassant pl 163)

La majuscule dans le pronom $O n$ est une marque du respect lorsque l'énoncé réfère à Dieu.

Pour terminer, nous montrons des modifications qui altèrent aussi bien le Dé que le discours du rapporteur.

\section{LA MISE EN ÉVIDENCE DU DÉ PAR DES AJOUTS}

Un facteur qui influe aussi sur la manifestation du Dé est l'étoffement qui introduit des caractéristiques nouvelles. Elle rend le Dé plus expressif et parlé. Voilà deux exemples :

\subsection{Intensification}

(12) Julien raviva ses répulsions en lui racontant gaiement [...]que le boulanger ayant entendu quelque bruit dans son four, la veille, qui n'était pas jour de cuisson, avait cru y surprendre un chat rôdeur et avait trouvé sa femme « qui n'enfournait pas du pain ». (Maupassant fr 148)

(12’) Julian opowiedział jej ze śmiechem [...] że ubiegłego wieczoru (a nie był to dzień wypieku) piekarz, słysząc jakieś szelesty w piecu myślał, że przyłapie tam bezpańskiego kota, a tymczasem przyłapał żonę, która „wcale nie była zajęta wsadzaniem chleba”. (Maupassant pl 118)

Le fragment entre guillemets est modifié par un intensifieur wcale 'pas du tout' qui fait écarter avec force l'idée de l'occupation habituelle de la femme du boulanger, et chercher une autre solution, suggérée par le rire grossier de Julien. 


\subsection{Répétition}

La répétition d'un mot suggère l'oral et le fait qu'il s'agit d'un mot anglais peut se comprendre comme une manifestation du snobisme ou de vanité :

(13 quoiqu'elle ne lui eût pas caché sa surprise qu'il habitât ce quartier qui devait être si triste et « qui était si peu smart pour lui qui l'était tant». (Proust fr, 196)

(13') w dzielnicy [...] tak mało smart, jak na niego, który jest wcieleniem smartu". (Proust pl 234)

\section{LA MISE EN ÉVIDENCE DU NARRATEUR}

Le DI est un cas du DR avec le contrôle apparent du narrateur (Short, Leech apud Taivalkoski-Shilov 2006 : 49). Ce contrôle peut devenir plus fort et favoriser une autre contextualisation du Dé. À notre avis, les modifications de ce type sont importantes pour établir les variantes d'un même texte dans le passage dans une autre langue (Adam 2005).

\subsection{L'image d'un narrateur qui maîtrise la variation diaphasique}

Le narrateur peut être présenté comme cultivé, par des équivalents recherchés d'un verbe de parole neutre :

(14) Dans ses poèmes, il parle du « mal princier » qui l'affecte et le ronge. (Makine fr 157)

(14') W swoich poematach młody człowiek opiewa „zło książęce”, które go niszczy. (Makine pl 141)

Le verbe parler est traduit par opiewać, un mot livresque 'vanter qq ou qch d'une manière belle et recherchée’ (SJP PWN). Le calque mal princier - zło ksiązęce, expression inhabituelle pour une expression familière choroba królów peut être considérée comme la recherche d'un style soigné, qui caractérise tout le fragment.

Le choix d'une conjonction livresque, p.ex. $i \dot{z}$, également peut construire une image du narrateur qui soigne son style :

(15) [il] déclara que « bien sûr, cette histoire ne l'avait pas empêché de connaître l'amour... et comment! [...] (Mauriac fr 846)

(15') oświadczył, ì̇ ,ta historia nie przeszkodziła mu oczywiście poznać miłości... i to jeszcze jak! [...] (Mauriac pl 163)

\subsection{L'image d'un narrateur qui maîtrise les opérations de liage}

Le traducteur peut modifier les énoncés de manière à mettre en évidence la compétence du narrateur à construire la continuité textuelle (Adam 2008 : 83), d'une manière plus diversifiée que dans le TD. Reprenons l'exemple (5) : 
(16/5) et madame Tuvache [...] déclara devant sa servante que madame Bovary se compromettait. (Flaubert fr 160)

(16/5') a pani Tuvache [...] orzekła nawet, i to przy służącej ('déclara même, et en plus devant la servante'), że doktorowa się kompromituje. (Flaubert E 111)

Les paroles de madame Tuvache sont mises en évidence par le cumul du marqueur nawet 'même', qui signale le renchérissement, et de l'expression $i$ to qui signale quelque chose comme notable et inattendu. Le cotexte du Dé, énoncé par le narrateur, crée l'attente d'un contenu important et influe beaucoup sur la compréhension du texte traduit.

CONCLUSION

La traduction du discours indirect mimétique du français vers le polonais, présentée sur un échantillon de textes littéraires montre plusieurs aspects intéressants quant à la présence plus ou moins marquée du Dé. On observe un facteur grammatical important de l'actualisation, le système temporel sans la concordance des temps. La réduction du démonstratif relève d'un usage collectif. Les termes de désignation sont une ressource liée avec les savoirs supposés des lecteurs. Les manières de parler des personnages sont difficiles à rendre lorsqu'elles reposent sur une inférence, sur l' évaluation de l'intonation ou de la longueur et de la structure de l'énoncé. Le lexique spécialisé d'un domaine est un marqueur lorsqu'il existe dans les deux langues. Les frontières du segment qui produit l'effet de réel (guilemets, italique) changent selon le traducteur. Ces cas exigent du lecteur une construction active des effets de sens.

Il est pertinent de tenir compte des modifications qui mettent en relief aussi bien le Dé que les énoncés du narrateur. Ceux-ci constituent le contexte de l'interprétation pour les paroles du personnage, et c'est la cohérence qui est un facteur supplémentaire de l'interprétation. La retraduction met en évidence la création d'un texte qui produit dans les énoncés au DI l'effet de réel variable (Dutka-Mańkowska 2012). La traduction s'avère « une forme privilégiée de l'interprétation» (Brzozowski 2011 : 37) et l'effet de réel est à lier à l'attitude active du lecteur.

\section{BIBLIOGRAPHIE:}

Adam Jean-Michel, 2005, Les sciences de l'établissement des textes et la question de la variartion, (in : ) Sciences du texte et analyse du discours. Enjeux d'une interdisciplinité, Jean-Michel Adam, Ute Heidmann (éds), Genève : Slatkine Érudition, 69-96.

Adam Jean-Michel, 2008, La linguistique textuelle. Introduction à l'analyse textuelle des discours, $2^{\mathrm{e}}$ éd., Paris : A. Colin.

Anscombre Jean-Claude, 1995, La théorie de topoï: rhétorique ou sémantique ? Hermès 15, 185-198.

Authier-Revuz Jacqueline, 1993, Repères dans le champ du discours rapporté, L'Information Grammaticale 56, 10-15.

AutHIER-Revuz Jacqueline, 2001, Le discours rapporté, (in: ) Grands repères culturels pour une langue : le français, Roberte Tomassone (ed.), Paris: Hachette Education, 192-201. 
Authier-Revuz Jacqueline, 2004, La Représentation du discours autre : Un champ multiplement hétérogène, (in : ) Le discours rapporté dans tous ses états, Juan Manuel López Muñoz, Sophie Marnette, Laurence Rosier (dir.), Paris : L'Harmattan, 35-53.

BAKhtine Michail, 1978, Esthétique et théorie du roman, trad. Daria Ollivier, Paris : Gallimard.

Brzozowski Jerzy, 2011, Stanać po stronie tłumacza. Zarys poetyki opisowej przektadu, Kraków : Wydawnictwo Uniwersytetu Jagiellońskiego.

Coulmas Florian, 1986, Reported Speech: Some General Issus, (in:) Direct and Indirect Speech, Florian Coulmas (ed.), Berlin : Mouton de Gruyter, 1-28.

De Mattia-Viviès Monique, 2006, De la porosité des formes du discours rapporté aux cas de déconnection forme/sens dans l'univers du récit, (in :) Discours rapportés. Approches(s) linguistique(s) et/ou traductologique(s), Catherine Delesse (dir.), Arras: Artois Presses Université, 29-52.

De MatTia-Viviès Monique, 2010, Du discours rapporté mimétique aux formes intrinsèquement hybrides, Anglophonia 28, 151-180.

DutKa-MaŃKOWSKa Anna, 2012, Le discours autre dans la retraduction de Madame Bovary en polonais, (in : ) Pluralité des cultures : chances ou menaces?, Alicja Kacprzak, Mirosław Gajos (éds.), Łask : Oficyna Wydawnicza LEKSEM, 117-127.

DutKa-MaŃKowska Anna, 2015, Des effets d'oralité dans des tertes littéraires et leur traduction du français vers le polonais, (in :) Regards sur l'oral et l'écrit, Krystyna Wróblewska-Pawla, Jolanta Sujecka-Zając, Elżbieta Pachocińska (dir.), Warszawa, Wydawnictwa Uniwersytetu Warszawskiego, $34-40$.

DutKa-MaŃKowska Anna, 2016, Le démonstratif comme marqueur d'un point de vue subjectif en français et sa traduction en polonais, (in :) Les émotions et les valeurs dans la communication I. Découvrir l'univers de la langue, Anna Krzyżanowska, Katarzyna Wołowska (éds.), Frankfurt a.M. : P. Lang, 35-44.

DutKa-MaŃKowsKa Anna, Kostro Monika (dir.), 2017, Le discours représenté dans les genres écrits et oraux, Lublin : Werset.

Jonasson Kerstin, 2002, Formes du discours rapporté dans Une vie de G. de Maupassant : citation et reformulation, Romansk Forum 16-2, 517-526.

LANE-Mercier Gillian, 1989, La parole romanesque, Ottawa-Paris : Les Presses de l'Université d'Ottawa.

LeEman Danielle, 2004, Les déterminants du nom en français. Syntaxe et sémantique, Paris : PUF.

Maingueneau Dominique, 1994, L'énonciation en linguistique française, Paris : Hachette Supérieur.

Poncharal Bruno, 2003, La représentation des paroles au discours indirect libre en anglais et en français, Paris : Ophrys.

Rabatel Alain, 2008, Point de vue et narration dans La Mère Sauvage de Maupassant: regard froid, passions chaudes, Synergies Pays Riverains de la Baltique 5, 105-128.

RIEGEL Martin et al. 2009, Grammaire méthodoque du français. Édition revue et augmentée, Paris : PUF.

Rosier Laurence, 1999, Le discours rapporté. Histoire, théories, pratiques, Paris: Duculot.

Rosier Laurence, 2006, Discours rapporté et diversité des langues : quelques problèmes relatifs à la polyphonie et au plurilinguisme, (in :) Discours rapportés. Approches(s) linguistique(s) et/ou traductologique(s), Catherine Delesse (dir.), Arras : Artois Presses Université, 11-28.

RosiER Laurence, 2008, Le discours rapporté en français, Paris : Ophrys.

TAIVALKoSki-Shilov Kristina, 2006, La tierce main. Le discours rapporté dans les traductions françaises de Fielding au XVIII siècle, Arras : Artois Presses Université.

Tomaszkiewicz Teresa, 1988, Étude comparative de quelques indices du concept d'énonciation en français et en polonais, Poznań : Wydawnictwo Naukowe UAM.

Wolf Nelly, 2016, Proses du Monde, COnTEXTES 18, DOI : 10.4000/contextes.6244. 


\section{SOURCES DES EXEMPLES :}

ERnAux Annie (1983), La place, Paris : Gallimard.

Ernaux Annie (1989), Miejsce, thum. Iwona Badowska, Warszawa : PIW.

Flaubert Gustave (2014), Madame Bovary, préface Anne Princen, Paris : Flammarion.

Flaubert Gustave (1955/2008), Pani Bovary, tłum. Anna Micińska, Kraków : Zielona Sowa.

Flaubert Gustave (2014), Pani Bovary. Z obyczajów prowincji, thum. i komentarze Ryszard Engelking, wyd. 2 zmienione, Warszawa : Sic!

Makine Andrei (1998), Le crime d'Olga Arbélina, Paris : Mercure de France.

MaKIne Andrei (1999), Zbrodnia Olgi Arbeliny, thum. Małgorzata Hołdyńska, Warszawa : Czytelnik.

Maupassant Guy de (1974), Une vie, éd. P. Cogny, Paris : Garnier - Flammarion.

Maupassant Guy de (1985), Historia pewnego życia, thum. Maria Kreczkowska, Warszawa : Zrzeszenie Księgarstwa.

MAURIAC François (1978), Le désert de l'amour, (in :) Oeuvres romanesques et théâtrales complètes, J. Petit (éd.), Paris : Gallimard, Bibliothèque de la Pléïade.

MAuriac François (1958), Pustynia miłości, thum. S. Zgórska, Warszawa : IW PAX.

Proust Marcel (1954), À la recherche du temps perdu, I, Du côté de chez Swann, Paris : Gallimard, Bibliothèque de la Pléïade.

Proust Marcel (2013), W stronę Swanna, tłum. T. Żeleński (Boy), Warszawa : MG.

Dictionnaires :

Słownik języka polskiego PWN, https://sjp.pwn.pl/

Lexilogos, http://www.lexilogos.com/francais_langue_dictionnaires.htm 